\title{
Consensus Guidelines for the Management of Patients with Digestive Neuroendocrine Tumors - Well-Differentiated Jejunal-Ileal Tumor/Carcinoma
}

\author{
Barbro Eriksson $^{a}$ Günter Klöppel ${ }^{b}$ Eric Krenning ${ }^{c}$ Hakan Ahlman ${ }^{d}$ \\ Ursula Plöckinger ${ }^{e}$ Bertram Wiedenmann ${ }^{e}$ Rudolf Arnold $^{f}$ \\ Christoph Auernhammer $^{g}$ Meike Körner ${ }^{\text {h }}$ Guido Rindi $^{i}$ \\ Stefan Wildi ${ }^{j}$ all other Frascati Consensus Conference participants ${ }^{1}$ \\ a Department of Medical Sciences, Endocrine Oncology Unit, University Hospital, Uppsala, Sweden; \\ ${ }^{\mathrm{b}}$ Department of Pathology, University of Kiel, Germany; ' Erasmus MC, Rotterdam, The Netherlands; \\ ${ }^{d}$ Institute for Surgical Sciences, Department of Surgery, Sahlgrenska sjukhuset, Gothenburg, Sweden; \\ ${ }^{e}$ Charité Universitätsmedizin, Campus Virchow-Klinikum, Department of Hepatology and Gastroenterology, \\ Berlin, ${ }^{f}$ Wittelsbacherstrasse 6, ${ }^{9}$ Medizinische Klinik II, Grosshadern Klinikum der Ludwig-Maximilians-Universität

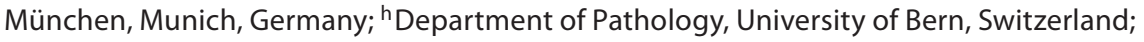 \\ 'Department of Pathology, University of Parma, Italy; ${ }^{j}$ University Hospital of Zürich, Department of Visceral and \\ Transplantation Surgery, Zürich, Switzerland
}

\section{Introduction}

\section{Epidemiology and Clinicopathological Features}

Neuroendocrine tumors of the lower jejunum and ileum account for $23-28 \%$ of all gastrointestinal endocrine tumors and incidence rates of $0.28-0.8$ per 100,000 population per year have been reported [1, 2]. Most of these tumors are well-differentiated and have an indolent course. As a consequence of the long delay between onset of symptoms and final diagnosis, many patients have advanced disease at the time of diagnosis. These tumors occur with equal frequency in males and females with an

B. Eriksson wrote the paper, which was revised by B. Wiedenmann, according to the comments and suggestions from G. Klöppel, E. Krenning, H. Ahlman, and U. Plöckinger; they and the other authors listed in alphabetical order equally contributed to the preparation of the Guidelines. age peak in the 6th and 7th decades of life [1]. The frequency of multicentricity lies between 26 and $30 \%[3,4]$ and $15-29 \%$ of tumors are associated with other non-carcinoid malignancies [5-7]. The WHO classification subdivides jejunal-ileal tumors, similar to other gastroenteropancreatic endocrine tumors, into three general categories [8]: (1) well-differentiated endocrine tumor (carcinoid) of benign behavior (confined to the mucosasubmucosa, non-angioinvasive, $<1 \mathrm{~cm}$ in size) or uncertain behavior (non-functioning, confined to mucosasubmucosa, $>1 \mathrm{~cm}$ in size, or angioinvasive); (2) well-differentiated endocrine carcinoma (malignant carcinoma) with low-grade malignant behavior, deeply invasive (muscularis propria or beyond) or with metastases, and (3) poorly-differentiated endocrine carcinoma (small-cell carcinoma), high-grade malignant.

\footnotetext{
1 See list at the end of the paper.
}

\section{KARGER}

Fax +41613061234 E-Mail karger@karger.ch www.karger.com (c) 2007 S. Karger AG, Basel

$0028-3835 / 08 / 0871-0008 \$ 24.50 / 0$

Accessible online at:

www.karger.com/nen
Barbro Eriksson

Department of Medical Sciences, Endocrine Oncology Unit, Entrance 40

University Hospital, SE-75185 Uppsala (Sweden)

Tel. +4618 611 2716, Fax +4618 553943

E-Mail barbro.eriksson@medsci.uu.se 


\section{Minimal Consensus Statement on Epidemiology and Clinicopathological Features}

The clinical incidence for small intestinal endocrine tumors is considerably lower than the incidence at autopsy (1/150) [9]. Clinical incidence is probably higher than stated earlier in the literature. The incidence for jejunal-ileal tumors in the panSEER registry (1973-1999) is $26 \%$ among gastrointestinal carcinoids [1]. The average age at diagnosis for patients with small intestinal carcinoids is 65.4 years and comparison of registries suggests an overall increase in the average age at diagnosis [1]. According to the literature there is no gender preference, whereas there are some racial differences. Black Americans have a slightly higher risk than Caucasians (1.09 corrected ratio) of developing jejunal-ileal tumors.

\section{Clinical Presentation}

Non-Functioning Tumors. Asymptomatic endocrine tumors in the distal small intestine are discovered while searching for a primary in patients with newly discovered liver metastases from an endocrine tumor or incidentally during colonoscopy or intubation of the terminal ileum. At the time of diagnosis, the tumors are commonly $>2 \mathrm{~cm}$ and have invaded the muscularis propria and also metastasized to regional lymph nodes. Typical symptoms include intermittent abdominal discomfort misinterpreted as irritable bowel disease for many years. Peritumoral fibrosis can lead to intestinal obstruction and by adhesions of intestinal loops or luminal stricture, which may lead to ileus [10]. Some patients may develop nonsecretory diarrhea due to bacterial overgrowth. Furthermore, fibrosis around mesenteric metastases can cause fixation of the ileal mesentery to the retroperitoneum with fibrous bands obstructing the small intestine and transverse colon. This desmoplastic reaction may culminate in small bowel ischemia or hydronephrosis.

Functioning Tumors. Up to 18\% of patients with liver metastases due to an endocrine tumor of the jejunum-ileum present the carcinoid syndrome [11]. Signs and symptoms of the carcinoid syndrome can include one or any of the following: flushing, diarrhea, carcinoid heart disease (CHD), and intermittent bronchoconstriction. Abdominal pain due to the desmoplastic reaction surrounding the primary and mesenteric lymph node metastases can also be present in these patients.

Carcinoid crisis is a severe and potentially fatal exacerbation of hormonal symptoms often provoked by anesthesia or invasive procedures, such as surgery. The clinical picture includes flushing, hypo- or hypertension, diarrhea, severe bronchospasm and cardiac arrhythmias.

\section{Minimal Consensus Statement on Clinical Presentation}

The consensus was that the rate of functionality and presence of the carcinoid syndrome in this patient group is about $20-30 \%$, i.e. higher than previously stated. It was also stressed that small bowel ischemia could be another cause of diarrhea and pain besides hormone-related diarrhea. In the literature, flushing is reported to be a more frequent presenting symptom (in $90 \%$ of patients with the carcinoid syndrome) than diarrhea (80\%), but according to clinical experience the rate is roughly the same. It was emphasized the atypical carcinoid syndrome due to histamine by persecretion is not seen in midgut jejunal-ileal endocrine tumors. The carcinoid syndrome is usually seen in patients with liver metastases (in 95\% of patients), but excess tachykininserotonin production from retroperitoneal metastases or ovarian tumors/metastases can bypass the liver and enter the systemic circulation and cause the typical carcinoid syndrome (in $5 \%$ of patients).

\section{Prognosis}

The prognosis of tumors of the jejunum-ileum is generally unfavorable if compared to that of other gastrointestinal endocrine tumors, since they have a tendency to spread via regional lymph nodes to the liver [3, 10]. Survival of endocrine jejunal-ileal tumors correlates closely with the stage of the disease at presentation with a 5 -year survival of $65 \%$ in patients with localized or regional disease and $36 \%$ in those with distant metastases $[1,3,10$, 12]. Patients with slow-growing well-differentiated tumors and those with a low Ki-67 live longer than those with a more rapidly growing well-differentiated with a high Ki-67 [13].

\section{Minimal Consensus Statement on Prognosis}

In contrast to other gastrointestinal endocrine tumors, tumors of the jejunum-ileum of comparable size can behave very aggressively [14]. According to the literature, only $23 \%$ of patients having undergone apparently curative resection were free of disease after 25 years [15]. Regional lymph node metastases are present at the time of diagnosis in $36-39 \%$ and non-localized disease is evident in $64.1 \%$ of the patients [1]. For localized tumors of the small intestine, the 5 -year survival rate is $65-75 \%$ [1, $7,16]$; for regional disease the corresponding figure is $64-73 \%$ [1], whereas for non-localized tumors the 5 -year survival is $50 \%$ according to the SEER registry [1]. The 5-year survival rate of patients with hepatic tumor spread is $18-32 \%[1,7,10]$. If all stages are combined, the 5 -year survival rate of neuroendocrine tumors of jejunum-ileum is $60.5 \%$ [1]. Whether extensive disease leads to poorer survival is not known but the presence of the carcinoid syndrome has been described to decrease survival. The 5 -year survival in these patients is $21 \%$ [17], and the median survival is 38 months from the first flush and 23 months from the detection of elevated urinary 5-hydroxyindoleacetic acid [18]. 
Fig. 1. Diagnostic algorithm for jejunalileal tumors.

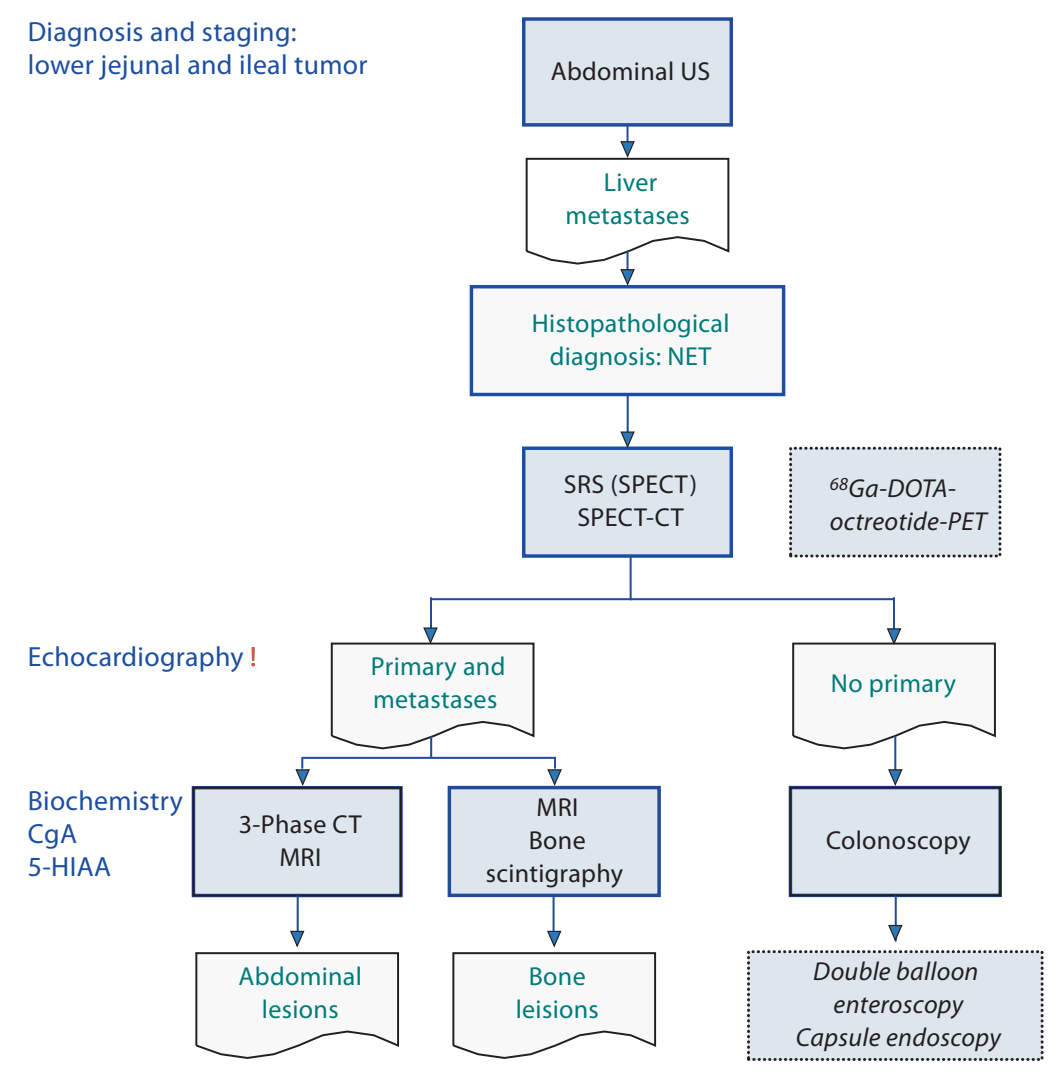

The prevalence of CHD in patients with the carcinoid syndrome is $30-65 \%[19,20]$. Right-sided heart failure, caused by $\mathrm{CHD}$ rather than tumor progression, was the cause of death in $30-50 \%$ of patients [19-21]. Besides high age and elevated 5-HIAA levels, tricuspid regurgitation was the most significant predictor of death [22].

Even though the age at diagnosis did not decrease, the survival rates among these patients are improving, which may be attributed to a multimodal approach with more aggressive surgery with removal of primary tumors and solitary liver metastases, medical therapy, and other new therapeutic options. The application of a new Tumor Node Metastases (TNM) classification for small intestinal endocrine tumors should prove to be an important prognostic tool and within the new classification, it may be prognostically valuable to distinguish between different M-stages.

\section{Diagnostic Procedures (fig. 1)}

\section{Imaging}

The standard imaging procedures for endocrine tumors of the lower jejunum-ileum include abdominal ultrasonography, contrast-enhanced helical CT or MRI of the abdomen in combination with somatostatin receptor scintigraphy (SRS), endoscopy, echocardiography, bone scan or spine MRI to prove bone metastases if SRS is negative.

In most patients with a metastasized jejunal-ileal carcinoid, transabdominal ultrasonography is the initial imaging procedure disclosing the presence of liver metastases. The technique is operator-dependent. Sensitivity and specificity have been variable. New contrast media have improved sensitivity and specificity (around 95\%). Ultrasound may be used to guide percutaneous coarse-needle biopsies for histopathological diagnosis (flowchart in fig. 1). 
After confirming the histopathological diagnosis, SRS with SPECT, which is positive in $80-90 \%$ of patients with small intestinal endocrine tumors, is a routine investigation for staging of the disease [23]. It may detect the primary tumor or regional lymph node metastases and additional metastatic lesions in the lung, breast, bone or brain. There are some limitations with regard to the spatial resolution $(<1 \mathrm{~cm})$ and negativity in lesions lacking the expression of sst 2 and sst5. Recently, positron emission tomography (PET) with ${ }^{68} \mathrm{Ga}$-labeled somatostatin analogs has been introduced as an alternative method in some centers [24].

In case the patient presents both a primary tumor and liver metastases, a three-phase high-resolution CT or MRI with contrast enhancement, which are complementary procedures with a similar sensitivity in detecting endocrine lesions in the abdomen [25-27], should be performed of positive areas to estimate the size of the lesions, determine the relationship to blood vessels, demonstrate surrounding desmoplastic reactions of mesenteric lymph nodes and primaries and, hence, judge the resectability of abdominal disease. A mesenteric tumor with radiating densities is considered pathognomonic for a mesenteric midgut metastasis.

Whereas liver metastases are easily detected by US/ CT/MRI or SRS, localization of the primary within the distal small bowel may be difficult. Colonoscopy can identify primary tumors in the distal ileum or the ileocecal valve. Barium enema or enteroclysis of the small intestine are rarely indicated. Instead new methods, such as capsule endoscopy or double balloon enteroscopy are promising.

Standard PET with ${ }^{18} \mathrm{~F}$-deoxyglucose (FDG) is not efficient in well-differentiated endocrine tumors but recently the use of PET with specific tracers for neuroendocrine tumors with ${ }^{11} \mathrm{C}-5$-hydroxytryptophan (HTP) or ${ }^{18} \mathrm{~F}$-dihydroxyphenylalanine (DOPA) has shown promising results, particularly in classical midgut carcinoid with sensitivities exceeding $90 \%[28,29]$. PET with these tracers is better for detection of small primary tumors and lymph node metastases than SRS or CT/ MRI.

If bone metastases are indicated by SRS, MRI is recommended to estimate the tumor mass within the skeleton and indicate the risk of fractures. If SRS is negative, bone scan can be performed.

Echocardiography is mandatory in patients with the carcinoid syndrome to confirm or exclude coexisting $\mathrm{CHD}$ and to judge the severity of the manifestation.

\section{Minimal Consensus Statement on Imaging}

In the search for a primary tumor, colonoscopy should be performed particularly in the context of synchronous neoplastic disease. Double balloon enteroscopy will probably have a role in the future but the method needs further validation. Capsule endoscopy can be useful after enteroclysm has failed to detect the intestinal primary tumor(s). Capsule endoscopy has a similar diagnostic yield as SRS and the advantage of being able to differentiate between intestinal and mesenterial localization [30, 31]. Endoscopic ultrasonography, on the other hand, has no place in this setting.

Fusion imaging with SRS plus SPECT/CT is preferred since it provides a more accurate morphological localization. If SRS is negative, ${ }^{18} \mathrm{~F}-\mathrm{DOPA}-\mathrm{PET}$ or ${ }^{11} \mathrm{C}-5$-HTPPET may be considered. ${ }^{68} \mathrm{Ga}$-DOTA-octreotide-PET, which does not require an in-house cyclotron, is (not yet for sale/approved by EMEA/FDA; similar to the other PET agents except FDG), however, accessible in very few research centers, and has been demonstrated to detect $30 \%$ more lesions than SRS [24].

\section{Laboratory Tests}

A specific constellation of symptoms should be followed up by performance of specific biochemical tests. Chromogranin A may serve as a sensitive marker in functioning and non-functioning tumors of the jejunum-ileum. However, its specificity is rather low in midgut neuroendocrine tumors. There is a correlation between the chromogranin A levels and prognosis [32]. It should be mentioned that available assays for chromogranin A differ in sensitivity due to different antibody specificities [33]. Endocrine tumors of the jejunum-ileum produce serotonin and elevated 24-hour urinary 5-HIAA levels. 5HIAA has a sensitivity of $73 \%$ and a specificity of $100 \%$ in predicting the presence of a carcinoid in the midgut area [33, 34]. However, chromogranin A is more sensitive, $87 \%$ [32], and can signal recurrences early in patients with a small tumor burden [35]. False positive chromogranin A levels can be found in patients medicating with proton pump inhibitors, atrophic gastritis, renal impairment, or inflammatory bowel disease. 5-HIAA should be collected with strict dietary restrictions to avoid false positive levels.

\section{Minimal Consensus Statement on Laboratory Tests}

The minimally required biochemical tests include chromogranin A and HIAA. They should be performed at first visit and then for follow-up. 


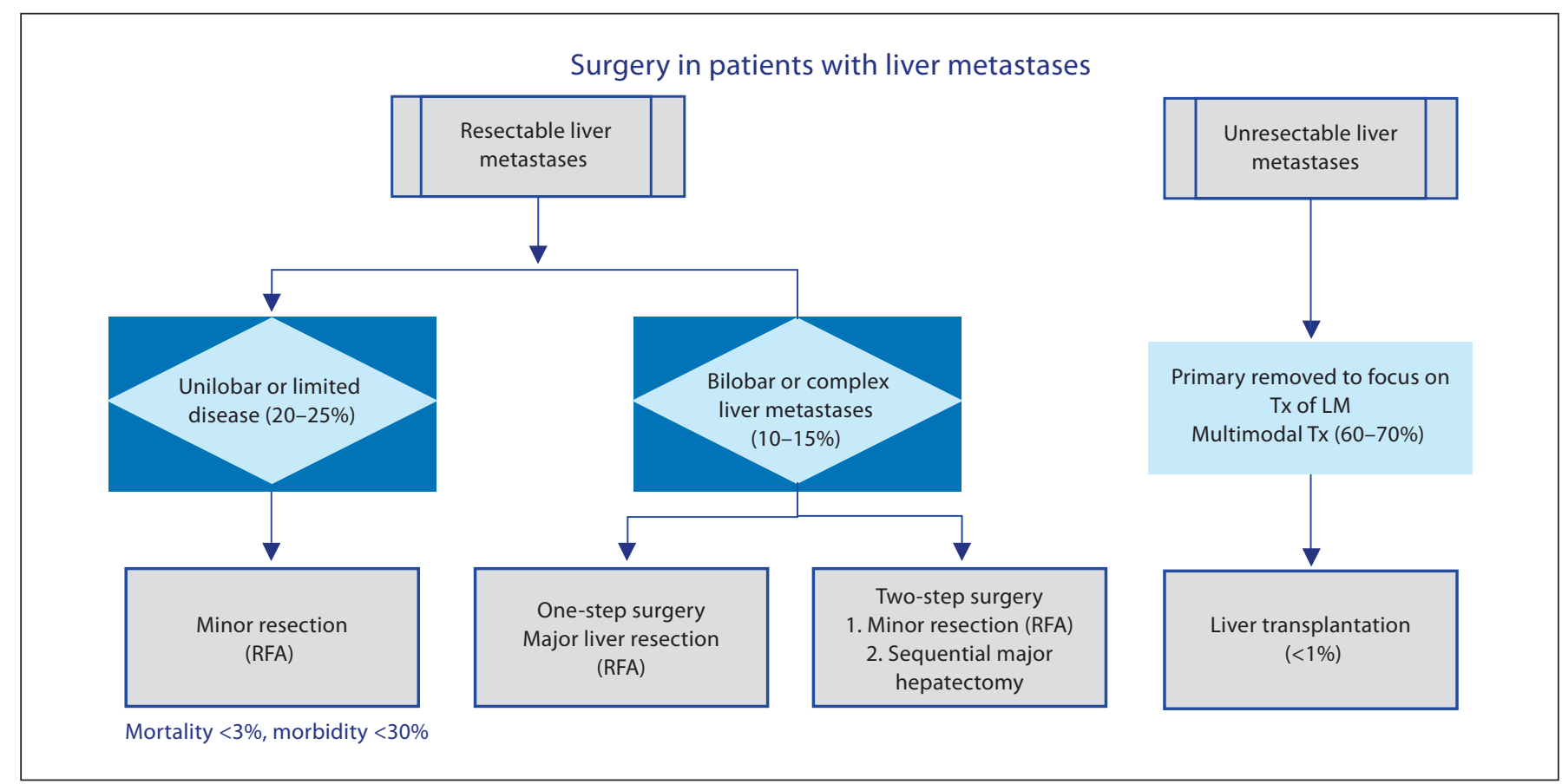

Fig. 2. Diagnostic algorithm for surgery in patients with liver metastases.

\section{Pathology and Genetics}

Pathological diagnosis is mandatory in all cases and usually obtained on ultrasonography-guided liver biopsies or biopsies from surgery. Pathological diagnosis of jejunal-ileal tumors is achieved using hematoxylin-eosin staining, immunohistochemical staining with chromogranin A and synaptophysin [36]. Determination of mitotic index and calculation of Ki- 67 index by immunohistochemistry is mandatory. The tumors should be classified according to the WHO system [8], knowing that the vast majority of tumors belong to WHO group 2 .

\section{Minimal Consensus Statement on Pathology and Genetics}

\section{Histopathology}

Histology is always necessary to establish diagnosis. Cytology may be helpful but is insufficient. The minimal ancillary tests to support the histological diagnosis include immunohistochemistry for chromogranin A, synaptophysin, and optionally serotonin. Both the mitotic count in $10 \mathrm{HPF}\left(2 \mathrm{~mm}^{2}\right.$, at least 40 fields (at $40 \times$ magnification) evaluated in areas of highest mitotic density, and the Ki-67 index (MIB1 antibody; \% of 2,000 cells in areas of highest nuclear labeling) [37]. The histopathology report should allow for a correct classification according to the current WHO criteria. In the future, it should also provide information for a correct TNM-classification and grading (see be- low). Immunohistochemistry for p53 and sst 2 is not routinely recommended, with the exception of staining for sst2A if SRS is not available.

\section{Genetics}

Although a familiar clustering of midgut neuroendocrine tumors $[38,39]$ appears to exist, the genetic background has not been elucidated and there is no indication to perform genetic counseling, germline or somatic DNA testing.

\section{Summary of TNM Proposal}

A proposal for a TNM classification for tumors of the lower jejunum and ileum, where T1-4 describes size of the tumor (with T2 tumors $>1 \mathrm{~cm}$ ) and the depth of involvement of the intestinal wall, was discussed. Stage I comprises T1 tumors with limited growth. Stage II identifies tumors that are larger in size or more invasive, i.e. T2 or T3, but without metastases. Stage III encompasses tumors invading surrounding structures (IIIa), T4, or the presence of regional lymph node metastases (IIIB), whereas stage IV indicates distant metastases. In the proposal, a grading system is also included, in which the G1 tumor category has a mitotic count $<2$ or $\mathrm{Ki}-67<2 \%$, G2 tumors a mitotic count 2-20 or Ki-67 2-20\%, and G3 tumors mitotic count $>20$ or Ki-67 $>20 \%$. The grading system and the WHO criteria are not exactly coherent with regard to mitotic count/Ki-67, since G3 tumors can belong to either 
group 2 tumors (well-differentiated carcinoma) or group 3 (poorly-differentiated carcinoma with Ki- $67>30 \%$ according to WHO). As a consequence of the proposal, the pathology report should include information about proliferation index, immunohistochemistry for chromogranin A, synaptophysin, tumor diameter and invasion. With multiple lesions, the largest diameter should be reported and for the N0 definition, a minimum of 12 lymph nodes should be assessed.

\section{Surgical Therapy (fig. 2)}

\section{Curative Surgical Therapy}

Surgery of Localized Disease in Jejunal-Ileal Tumors. Curative surgery should be aimed for in patients with endocrine tumors of the distal jejunum-ileum and localized disease. The size of the primary does not correspond to the metastatic propensity. Since tumor spread to lymph nodes and the liver can occur also in patients with small primary tumors, surgery of the primary should adhere to oncological principles. This involves clearance of lymph node metastases by dissection around the mesentery, aiming to preserve the vascular supply and to limit intestinal resection [40-45].

Surgery with Curative Intent of Jejunal-Ileal Carcinoids Metastatic to the Liver. Surgery has until now been therapy with a curative potential. Curative tumor resection, i.e. removal of the primary, regional lymph nodes, and resectable liver metastases, is possible in up to $20 \%$ of the patients [42, 46, 47, Ahlman, 2004, \#369]. Liver surgery includes metastasis enucleation, segmental resection, hemihepatectomy or extended hemihepatectomy. Intraoperative ultrasonography should be performed for detection of all liver metastases. Perioperative mortality is $<3 \%$ in most reports and postoperative 5 -year survival rate is $61 \%$ and even higher in some centers [46-53]. In contrast, in patients with midgut carcinoids and with liver metastases without surgical therapy, recent publications report a 5-year survival rate of $30 \%$ with a median survival of $3-4$ years $[7,46,47,51$, 54-57]. Curative intent in these studies was defined as the possibility of complete tumor resection. The comparison is, however, difficult, since most reports are from single institutions and no prospective randomized studies have been performed comparing medical therapy with surgery alone [11]. As a prerequisite for these extended procedures, mortality should be $<5 \%$ and morbidity $<30 \%$.

Well-Differentiated Jejunal-Ileal

Tumor/Carcinoma

\section{Minimal Consensus Statement on Curative Surgery}

Curative surgery is always recommended whenever feasible after careful symptomatic control of the clinical syndrome; the latter may be achieved by medical treatment (somatostatin analogs, interferon). Surgery of the primary should be performed as segmental resection with wide lymphadenectomy. In case of lymph node involvement around the superior mesenteric artery, high lymph node dissection is recommended. In cases with severe desmoplastic reaction around the artery, radical tumor resection may not be possible. Cholecystectomy is always recommended. Tumor multicentricity, which may occur in $20 \%$ of all cases, demonstrated by SRS, CT, intraoperative palpation and/ or endoscopy, does not change the indication for surgery. The outcome of surgery may be worse in cases of distant metastases other than the liver, as in cases with the so-called frozen mesenteric root and peritoneal carcinomatosis. After curative surgery, there is no indication for medical therapy other than pre- and perioperative somatostatin analog treatment to avoid carcinoid crisis.

\section{Palliative Surgery}

Primary Tumor and Liver Metastases. Cytoreductive surgery can be considered in all patients in whom $90 \%$ of the tumor can be safely removed $[49,51,53,58]$. Surgical intervention can be divided into resection of the primary with locoregional metastases or intraabdominal debulking, resection of liver metastases alone or synchronous resection of primary and liver metastases. Compared to patients with non-functional jejunal-ileal tumors, survival is reduced in patients with the carcinoid syndrome.

Primary Tumors Excluding Liver Metastases. Removal of the primary according to oncological criteria is indicated to prevent intestinal obstruction or ischemic complications due to a desmoplastic reaction of the mesenterium or compression of the mesenteric vein due to the tumor mass. As symptoms correlate with tumor mass, a reduction of tumor mass provides symptomatic relief in $70-100 \%$ of the patients. Intra-abdominal tumor resection (liver excluded) increased survival significantly from 69 to 130 months $[53,58]$. However, these data must be interpreted with caution, since surgery is mostly done in patients with less extensive disease and, in addition, most patients have received other therapies affecting the survival.

\section{Minimal Consensus Statement on Palliative Surgery}

Palliative surgery for patients with endocrine tumors of the jejunum-ileum has the objective to make liver metastases the only persisting problem. Patients suitable for palliative procedures are those presumed to benefit from tumor reduction performed in accordance with given guidelines. Palliative surgery should mainly be done for symptomatic reasons to facilitate for 
other therapeutic modalities, i.e. medical and radioactive treatment. The type of surgery should be individualized since no general approach can be given. If liver metastases require a minor resection, this can be done at the same procedure as the primary, otherwise it should be done in a second operation. In the palliative setting, medical therapy is usually required pre-, per-, and postoperatively.

\section{Medical Therapy}

\section{Biotherapy}

Biotherapy is defined as a therapeutic modality controlling hormonal hypersecretion syndromes and/or tumor growth with substances or pharmacological analogs thereof, occurring naturally in the body. There are numerous studies published in the literature, most of them with a small number of patients, but very few fulfill the criteria of evidence-based medicine, because of the low incidence of the tumor. Indeed, there are only a few studies on somatostatin analogs and none on interferon performed in a randomized, prospective, multicenter fashion, including patients with documented tumor progression.

Somatostatin Analogs. Somatostatin analogs effectively reduce hypersecretion-related symptoms in patients with the carcinoid syndrome. The antisecretory effect results in a reduction of biochemical markers in up to 40 $60 \%$ and a symptomatic improvement in $40-80 \%$ of patients [59-72]. The duration of the effect varies and can be limited due to tachyphylaxis or desensitization, which can be temporarily circumvented by an increase in dose. The antiproliferative effect of somatostatin analogs is modest, and partial or complete responses can be observed in fewer than $10 \%$ of the patients. However, stabilization of tumor growth occurs in $24-57 \%$ of patients with documented tumor progression before start of somatostatin analog therapy $[62,63,70,73,74]$. Hence, somatostatin analogs are clearly indicated for symptomatic relief in functioning jejunal-ileal endocrine tumors. Tolerance to somatostatin analogs and efficacy should be tested individually by initiating therapy with short-acting analogs. Thereafter, depot formulations, usually octreotide-LAR (20-30 mg) or Lanreotide Autogel every 4 weeks can be started and should be individually titrated. The efficacy of lanreotide and octreotide is comparable $[65,66,70]$. Minor initial side effects include abdominal discomfort, flatulence, and sometimes steatorrhea that usually subside $[61,66,67,75]$. In patients with steatorrhea, pancreatic enzyme replacement can help. More than $50 \%$ of patients have been reported to develop gall- stones, however, virtually all remain asymptomatic [75]. To prevent carcinoid crisis, somatostatin analogs should be given intravenously during anesthesia or other interventional procedures. Loperamide and morphine analogs may improve secretory diarrhea. Since diarrhea may have other causes than hormonal (bile acid loss, bacterial overgrowth) other options may be considered, such as cholestyramine and antibiotics.

\section{Minimal Consensus Statement on Somatostatin Analogs}

Somatostatin analog therapy is recommended as first-line medical treatment in functioning tumors. It provides symptomatic improvement in $70-80 \%$ of patients and stabilization of tumor growth in up to $50 \%$ of patients with varying duration. Whether somatostatin analogs should be used in non-functioning tumors is not established but ongoing studies should clarify this issue. SRS-positive tumors tend to respond better than SRSnegative tumors. At least one study on lanreotide-PR showed that quality of life was improved by treatment [61] but there is no apparent relation to the dose of somatostatin analogs.

As mentioned before, cholecystectomy is recommended during abdominal surgery to prevent the development of gallstones.

Steatorrhea may lead to malabsorption of vitamin D with reduced calcium absorption [75]. Patients on somatostatin analogs should also have vitamin $B_{12}$ levels monitored, since these levels may be reduced possibly due to inhibition of intrinsic factor. To control diarrhea, loperamide, cholestyramine (after ileocecal/ileal resection) or pancreatic enzyme substitution may be necessary.

Interferon. Interferon- $\alpha$ is given for the same indication as somatostatin analogs, with the exception of carcinoid crisis. Its effect on symptom control, though not as rapid, is comparable to somatostatin analogs. A biochemical and symptomatic response could be noted in up to $50 \%$ of patients, whereas partial tumor size responses could be demonstrated in 10-15\% [76-87]. The duration of response was 12-36 months. Due to more pronounced side effects, interferon is generally used as second-line therapy for symptomatic control. Interferon, usually recombinant interferon- $\alpha$, is given subcutaneously at 3-5 MU 3-5 times a week. A pegylated formulation, i.e. long-acting given once a week, is available but not yet registered. Minor side effects include flu-like symptoms (relieved by paracetamol), weight loss and fatigue. Major side effects include autoimmune reactions, depression and mental disturbances. Bone marrow toxicity is usually mild as is hepatotoxicity, which can be managed by dose adjustments. 


\section{Minimal Consensus Statement on Interferon Treatment}

Interferon treatment is recommended as second-line treatment of functioning tumors of the jejunum-ileum with a low proliferation rate. The dose should be individually titrated according to the leukocyte count and given at night for better tolerance. Similarly to somatostatin analogs the use of interferon in non-functioning tumors is not established, but future trials may clarify this issue.

Whether the combination of somatostatin analogs and interferon is more effective than the single drugs has not been established [70,88-90] but patients progressing on monotherapy of either drug may benefit from the addition of the other drug. As adjuncts to control diarrhea, loperamide, cholestyramine (after ileocecal/ileal resection) or pancreatic enzyme substitution may be indicated.

\section{Chemotherapy}

The results with systemic chemotherapy have been poor in patients with jejunal-ileal endocrine tumors. Single agents (5-FU, doxorubicin, DTIC) and combinations (streptozotocin + doxorubicin or 5-fluorouracil) generally produce responses in $<15 \%$ of patients $[15,17$, 91-93].

\section{Minimal Consensus Statement on Chemotherapy}

There is a general consensus that chemotherapy with agents available today is not recommended in this patient category. The only exception represents poorly-differentiated carcinomas.

\section{Peptide Receptor Radionuclide Therapy}

Most endocrine jejunal-ileal tumors express somatostatin receptors, especially subtype 2 (sst2). Targeting these receptors with radiolabeled somatostatin analogs may not only be used for imaging but also for radiotherapy. Since the early 1990s, different radiolabeled analogs have been used for tumor-targeted therapy [94-97]. For metastatic disease with evidence of expression of sst 2 as demonstrated by SRS, two still FDA- and EMEA-unapproved analogs for peptide receptor radionuclide therapy (PRRT) - ${ }^{90}$ Y-DOTA-octreotide [98-109] and ${ }^{177} \mathrm{Lu}-$ DOTA-octreotate (Lutate) [110-113] - show promising results. Phase I-II trials have shown symptomatic improvement in advanced cases of neuroendocrine tumors. Partial and minor responses and stabilization in patients with progressive disease at the start of treatment occurred in $12-35,12-14$, and $28-56 \%$, respectively. The most recent data indicate PR or CR in $28 \%$ of patients with a median time to progression of more than 36 months for Lutate [114].

These radiopharmaceuticals are only available in a few centers. The treatment has to be performed in collaboration with nuclear medicine units. Side effects are limited as long as radiation dose to the kidney and bone marrow are kept within dose limits; the use of kidney protection by co-infusion of amino acids (lysine and arginine) allows the administration of higher doses of the radiopharmaceuticals.

\section{Minimal Consensus Statement on PRRT}

PRRT is recommended in SRS-positive tumors in symptomatic patients refractory to medical treatment with inoperable disease. ${ }^{90} \mathrm{Y}$ - or ${ }^{177} \mathrm{Lu}$-coupled analogs should be employed.

\section{Carcinoid Heart Disease}

CHD develops in $40-50 \%$ of patients with the carcinoid syndrome $[19,20]$ and has been described to be the cause of death in $30-50 \%$ of patients [20,21]. It is characterized by plaque-like, fibrous endocardial thickening that principally involves the right side of the heart, causing retraction and fixation of the leaflets of the tricuspid and pulmonary valves as well as diminished right ventricular function [115]. Tricuspid regurgitation is a nearly universal finding. These valvular lesions will eventually lead to right-sided heart failure. The pathogenesis of the fibrosis in the right side of the heart has now been partly clarified by Gustafsson et al. [116], showing that serotonin plays a role. Several earlier studies have shown that HIAA and tachykinins are higher in those patients with CHD than in those without it [20].

Screening for CHD should be performed on a regular basis. If it develops, heart failure rather than metastatic disease may be the cause of death. Medical therapy for heart failure should be introduced when necessary and cardiac surgery with valve replacement (bioprosthesis) should be considered for patients, in whom control of hormonal symptoms and tumor growth has been achieved. Cardiac surgery should be performed before major liver surgery or liver embolization. 


\section{Minimal Consensus Statement on Carcinoid Heart Disease}

For patients with the carcinoid syndrome and CHD, transthoracic echocardiography should be performed annually. For timing of cardiac surgery with replacement of the tricuspid (and pulmonary) valves, brain natriuretic peptide measurements may be helpful, since they reflect the load on the right side of the heart [117]. The decision should be made in collaboration with an experienced cardiologist.

\section{Follow-Up}

For patients having undergone surgery with a curative intent, the schedule for follow-up should be every 6-12 months, with the exception of grade 3 tumors, which should be followed every 3 months. Minimal examinations include measurement of chromogranin A and 5HIAA and three-phasic CT. SRS should be done in suspected recurrences before any therapeutic decisions are made. The follow-up should be lifelong considering the epidemiological data indicating that after 25 years only $23 \%$ of patients are free of disease [15].

\section{List of Participants}

List of Participants of the 'Consensus Conference on the ENETS Guidelines for the Diagnosis and Treatment of Neuroendocrine Gastrointestinal Tumors, Part 2: Midgut and Hindgut Tumors' Held in Frascati (Rome, Italy), November 1-4, 2006

Martyn Caplin, Royal Free Hospital London, Centre for Gastroenterology, London NW3 2QG (UK); Gianfranco Delle Fave, Ospedale S. Andrea, Via di Grottarossa 1035, IT-00189 Rome (Italy); Diego Ferone, Departments of Internal Medicine and Endocrinological and Metabolic Sciences, University of Genoa, IT16132 Genoa (Italy); Peter Goretzki, Städtisches Klinikum Neuss, Lukaskrankenhaus, Chirurgische Klinik I, Preussenstr. 84, DE41456 Neuss (Germany); Rudolf Hyrdel, University Hospital Martin, II. Internal Medical Department, Kollarova 2 (Street), SK-03659 Martin (Slovakia); Robert Jensen, National Institutes of Health, Cell Biology Section, Building 10, Room 9C-193, Bethesda, Md. (USA); Gregory Kaltsas, G. Genimatas Hospital, Mesogion Ave., GR-10443 Athens (Greece); Fahrettin Keleştimur, Erciyes University Medical School, Department of Endocrinology and Metabolism, TR-38039 Kayseri (Turkey); Reza Kianmanesh, UFR Bichat-Beaujon-Louis Mourier, Service de Chirurgie Digestive, Hôpital Louis-Mourier, 178, rue des Renouillers, FR-92700 Colombes (France); Paul Komminoth, Institute for Pathology, Kantonsspital, CH-5404, Baden (Switzerland); Beata Kos-Kudła, Slaska Akademia Medyczna, Klinika Endokrynologii, ul. 3 Maja 13/15, PL-41800 Zabrze (Poland); Larry Kvols, H. Lee Moffitt Cancer Center/University of South Florida, Magnolia Drive 12902, Tampa, FL 33612 (USA); Dik Kwekkeboom, Depart- ment of Nuclear Medicine, Erasmus University Medical Center, Dr. Molewaterplein 40, NL-3015 GD Rotterdam (The Netherlands); José Manuel Lopes, IPATIMUP, Rua Dr. Roberto Frias SLN, PT-4200, Porto (Portugal); Riccardo Manfredi, Istituto di Radiologia, Policlinico GB Rossi, Piazzale LA Scuro, IT-37134 Verona (Italy); Anne Marie McNicol, Division of Cancer Sciences and Molecular Pathology, Pathology Department, Royal Infirmary, Castle Street, Glasgow G4 0SF (UK); Bruno Niederle, Universität Wien, Abteilung Chirurgie, Allgemeines Krankenhaus Wien, Währinger Gürtel 18-20, Ebene 3L, AT-1090 Vienna (Austria); Ola Nilsson, Department of Pathology, Sahlgrenska sjukhuset, SE-41345 Gothenburg (Sweden); George Nikou, 68, Plataion Street, GR-15235 Vrilissia/Athens (Greece); Kjell Öberg, Department of Medical Sciences, Endocrine Oncology Unit, University Hospital, SE-75185 Uppsala (Sweden); Juan O’Connor, Instituto Alexander Fleming, Crámer 1180, C1426ANZ, Buenos Aires (Argentina); Dermot O'Toole, Service de Gastroentérologie, Centre Hospitalier Universitaire d'Angers, FR-49000 Angers (France); Marianne Pavel, Charité Universitätsmedizin, Department of Internal Medicine, Division of Hepatology and Gastroenterology, Campus Virchow Klinikum, Augustenburger Platz 1, DE-13353 Berlin (Germany); Aurel Perren, Universitätsspital Zürich, Departement Pathologie, Histologielabor PATH F1, Schmelzbergstr. 12, CH-8091 Zürich (Switzerland); John Ramage, Department of Gastroenterology, North Hampshire Hospital, Aldermaston Rd, Basingstoke RG249NA, Hants (UK); Jens Ricke, Otto-von-Guericke-Universität Magdeburg, Klinik für Radiologie und Nuklearmedizin, Leipziger Str. 44, DE-29120 Magdeburg (Germany); Philippe Ruszniewski, Service de Gastroentérologie, Hôpital Beaujon, 100, Boulevard Général Leclerc, FR-92110 Clichy (France); Aldo Scarpa, Department of Pathology, University of Verona, Strada Le Grazie, 8, IT-37134 Verona (Italy); Jean-Yves Scoazec, Anatomie Pathologique, Hôpital Edouard-Herriot, Bâtiment 10, 3, place d'Arsonval, FR-69437 Lyon, Cedex 03 (France); Maria Isabel Sevilla Garcia, Hospital Virgen de la Victoria, C/Colonia Santa Ines s/n, ES-29010 Malaga (Spain); Thomas Steinmüller, DRK Kliniken Westend, Spandauer Damm 130, DE-14050 Berlin (Germany); Babs Taal, Netherlands Cancer Centre, Plesmanlaan 121, NL-1066 CX Amsterdam (The Netherlands); Marie-Pierre Vullierme, Service de Gastroentérologie, Hôpital Beaujon, 100, Boulevard Général Leclerc, FR-92110 Clichy (France); James C. Yao, The University of Texas M.D. Anderson Cancer Center, 1515 Holcombe Boulevard, Houston, TX 77030 (USA).

References

1 Modlin IM, Lye KD, Kidd M: A five-decade analysis of 13,715 carcinoid tumors. Cancer 2003;97:934-959.

2 Kulke MH, Mayer RJ: Carcinoid tumors. N Engl J Med 1999;340:858-868.

3 Burke AP, Thomas RM, Elsayed AM, Sobin LH: Carcinoids of the jejunum and ileum: an immunohistochemical and clinicopathologic study of 167 cases. Cancer 1997;79:10861093.

4 Peck JJ, Shields AB, Boyden AM, Dworkin LA, Nadal JW: Carcinoid tumors of the ileum. Am J Surg 1983;146:124-132. 
5 Modlin IM, Sandor A: An analysis of 8,305 cases of carcinoid tumors. Cancer 1997;79: 813-829.

6 Loftus JP, van Heerden JA: Surgical management of gastrointestinal carcinoid tumors. Adv Surg 1995;28:317-336.

7 Godwin JD 2nd: Carcinoid tumors. An analysis of 2,837 cases. Cancer 1975;36:560569.

8 Solcia E KG, Sobin LH, in collaboration with 9 pathologists from 4 countries: Histological Typing of Endocrine Tumors, ed 2. WHO International Histological Classification of Tumors. Berlin, Springer, 2000.

9 Berge T, Linell F: Carcinoid tumours. Frequency in a defined population during a 12year period. Acta Pathol Microbiol Scand [A] 1976;84:322-330.

10 Strodel WE, Talpos G, Eckhauser F, Thompson N: Surgical therapy for small-bowel carcinoid tumors. Arch Surg 1983;118:391-397.

11 Marshall JB, Bodnarchuk G: Carcinoid tumors of the gut. Our experience over three decades and review of the literature. J Clin Gastroenterol 1993;16:123-129.

12 McDermott EW, Guduric B, Brennan MF: Prognostic variables in patients with gastrointestinal carcinoid tumours. Br J Surg 1994; 81:1007-1009.

13 Arnold R, Rinke A, Klose KJ, et al: Octreotide versus octreotide plus interferon- $\alpha$ in endocrine gastroenteropancreatic tumors: a randomized trial. Clin Gastroenterol Hepatol 2005;3:761-771.

14 Oberg K: Chemotherapy and biotherapy in neuroendocrine tumors. Curr Opin Oncol 1993;5:110-120.

15 Moertel CG: Karnofsky memorial lecture. An odyssey in the land of small tumors. J Clin Oncol 1987;5:1502-1522.

16 Creutzfeldt W: Carcinoid tumors: development of our knowledge. World J Surg 1996; 20:126-131

17 Oberg K: The use of chemotherapy in the management of neuroendocrine tumors. Endocrinol Metab Clin North Am 1993;22: 941-952.

18 Ahlman H, Westberg G, Wangberg B, et al: Treatment of liver metastases of carcinoid tumors. World J Surg 1996;20:196-202.

19 Zuetenhorst JM, Bonfrer JM, Korse CM, Bakker R, van Tinteren H, Taal BG: Carcinoid heart disease: the role of urinary 5-hydroxyindoleacetic acid excretion and plasma levels of atrial natriuretic peptide, transforming growth factor- $\beta$ and fibroblast growth factor. Cancer 2003;97:1609-1615.

20 Norheim I, Oberg K, Theodorsson-Norheim E, et al: Malignant carcinoid tumors. An analysis of 103 patients with regard to tumor localization, hormone production, and survival. Ann Surg 1987;206:115-125.

21 Makridis C, Rastad J, Oberg K, Akerstrom G: Progression of metastases and symptom improvement from laparotomy in midgut carcinoid tumors. World J Surg 1996;20:900907.
22 Westberg G, Wangberg B, Ahlman H, Bergh $\mathrm{CH}$, Beckman-Suurkula M, Caidahl K: Prediction of prognosis by echocardiography in patients with midgut carcinoid syndrome. Br J Surg 2001;88:865-872.

23 Krenning EP, Kwekkeboom DJ, Bakker WH, et al: Somatostatin receptor scintigraphy with $\left[{ }^{111}\right.$ In-DTPA-D-Phe $\left.{ }^{1}\right]$ - and $\left.{ }^{[123}{ }^{12}-\mathrm{Tyr}^{3}\right]$ octreotide: the Rotterdam experience with more than 1,000 patients. Eur J Nucl Med 1993;20:716-731.

24 Hofmann M, Maecke H, Borner R, et al: Biokinetics and imaging with the somatostatin receptor PET radioligand ${ }^{68} \mathrm{Ga}$-DOTATOC: preliminary data. Eur J Nucl Med 2001;28: 1751-1757.

25 Ricke J, Klose KJ, Mignon M, Oberg K, Wiedenmann B: Standardisation of imaging in neuroendocrine tumours: results of a European delphi process. Eur J Radiol 2001;37: 8-17.

26 Kaltsas G, Rockall A, Papadogias D, Reznek $\mathrm{R}$, Grossman AB: Recent advances in radiological and radionuclide imaging and therapy of neuroendocrine tumours. Eur J Endocrinol 2004;151:15-27.

27 Bader TR, Semelka RC, Chiu VC, Armao DM, Woosley JT: MRI of carcinoid tumors: spectrum of appearances in the gastrointestinal tract and liver. J Magn Reson Imaging 2001;14:261-269.

28 Orlefors H, Sundin A, Garske U, et al: Wholebody ${ }^{11} \mathrm{C}-5$-hydroxytryptophan positron emission tomography as a universal imaging technique for neuroendocrine tumors: comparison with somatostatin receptor scintigraphy and computed tomography. J Clin Endocrinol Metab 2005;90:3392-3400.

29 Koopmans KP, de Vries EG, Kema IP, et al: Staging of carcinoid tumours with ${ }^{18} \mathrm{~F}$-DOPA PET: a prospective, diagnostic accuracy study. Lancet Oncol 2006;7:728-734.

30 Van Tuyl SA, van Noorden JT, Timmer R, Stolk MF, Kuipers EJ, Taal BG: Detection of small-bowel neuroendocrine tumors by video capsule endoscopy. Gastrointest Endosc 2006;64:66-72.

31 Swain P: Wireless capsule endoscopy. Gut 2003;52(suppl 4):48-50.

32 Oberg K, Stridsberg M: Chromogranins as diagnostic and prognostic markers in neuroendocrine tumours. Adv Exp Med Biol 2000; 482:329-337.

33 Ardill JE, Erikkson B: The importance of the measurement of circulating markers in patients with neuroendocrine tumours of the pancreas and gut. Endocr Relat Cancer 2003; 10:459-462.

34 Feldman JM, O’Dorisio TM: Role of neuropeptides and serotonin in the diagnosis of carcinoid tumors. Am J Med 1986;81:41-48.

35 Eriksson B, Oberg K: Peptide hormones as tumor markers in neuroendocrine gastrointestinal tumors. Acta Oncol 1991;30:477483.
36 Hamilton SR AL: Tumors of the digestive system. Pathology and Genetics. WHO Classification of Tumours. Lyon, ARC Press, 2000.

37 Rindi G, Kloppel G, Alhman H, et al: TNM staging of foregut (neuro)endocrine tumors: a consensus proposal including a grading system. Virchows Arch 2006;449:395-401.

38 Hemminki K, Li X: Incidence trends and risk factors of carcinoid tumors: a nationwide epidemiologic study from Sweden. Cancer 2001;92:2204-2210.

39 Hemminki K, Li X: Familial carcinoid tumors and subsequent cancers: a nationwide epidemiologic study from Sweden. Int J Cancer 2001;94:444-448.

40 Akerstrom G, Makridis C, Johansson H: Abdominal surgery in patients with midgut carcinoid tumors. Acta Oncol 1991;30:547553.

41 Rothmund M, Kisker O: Surgical treatment of carcinoid tumors of the small bowel, appendix, colon and rectum. Digestion 1994; 55(suppl 3):86-91.

42 Ahlman H, Wangberg B, Jansson S, et al: Interventional treatment of gastrointestinal neuroendocrine tumours. Digestion 2000; 62(suppl 1):59-68.

43 Makridis C, Oberg K, Juhlin C, et al: Surgical treatment of mid-gut carcinoid tumors. World J Surg 1990;14:377-384

44 Norton JA: Surgical management of carcinoid tumors: role of debulking and surgery for patients with advanced disease. Digestion 1994;55(suppl 3):98-103.

45 Goede AC, Winslet MC: Surgery for carcinoid tumours of the lower gastrointestinal tract. Colorectal Dis 2003;5:123-128.

46 Frilling A, Rogiers X, Malago M, Liedke OM, Kaun M, Broelsch CE: Treatment of liver metastases in patients with neuroendocrine tumors. Langenbecks Arch Surg 1998;383:6270.

47 Lehnert T, Knaebel HP: Diagnosis and therapy of liver metastases of neuroendocrine tumors (in German). Chirurg 1997;68:122131.

48 Chen H, Hardacre JM, Uzar A, Cameron JL, Choti MA: Isolated liver metastases from neuroendocrine tumors: does resection prolong survival? J Am Coll Surg 1998;187:8893.

49 Que FG, Nagorney DM, Batts KP, Linz LJ, Kvols LK: Hepatic resection for metastatic neuroendocrine carcinomas. Am J Surg 1995; 169:36-43.

50 Sarmiento JM, Heywood G, Rubin J, Ilstrup DM, Nagorney DM, Que FG: Surgical treatment of neuroendocrine metastases to the liver: a plea for resection to increase survival. J Am Coll Surg 2003;197:29-37.

51 Chamberlain RS, Canes D, Brown KT, et al: Hepatic neuroendocrine metastases: does intervention alter outcomes? J Am Coll Surg 2000;190:432-445. 
52 Norton JA, Warren RS, Kelly MG, Zuraek $\mathrm{MB}$, Jensen RT: Aggressive surgery for metastatic liver neuroendocrine tumors. Surgery 2003;134:1057-1065.

53 Soreide O, Berstad T, Bakka A, et al: Surgical treatment as a principle in patients with advanced abdominal carcinoid tumors. Surgery 1992;111:48-54.

54 Moertel CG, Sauer WG, Dockerty MB, Baggenstoss $\mathrm{AH}$ : Life history of the carcinoid tumor of the small intestine. Cancer 1961;14: 901-912.

55 Dousset B, Saint-Marc O, Pitre J, Soubrane O, Houssin D, Chapuis Y: Metastatic endocrine tumors: medical treatment, surgical resection, or liver transplantation. World J Surg 1996;20:908-915.

56 Chung MH, Pisegna J, Spirt M, et al: Hepatic cytoreduction followed by a novel long-acting somatostatin analog: a paradigm for intractable neuroendocrine tumors metastatic to the liver. Surgery 2001;130:954-962.

57 Wangberg B, Westberg G, Tylen U, et al: Survival of patients with disseminated midgut carcinoid tumors after aggressive tumor reduction. World J Surg 1996;20:892-899.

58 McEntee GP, Nagorney DM, Kvols LK, Moertel CG, Grant CS: Cytoreductive hepatic surgery for neuroendocrine tumors. Surgery 1990;108:1091-1096.

59 Eriksson B, Oberg K: Summing up 15 years of somatostatin analog therapy in neuroendocrine tumors: future outlook. Ann Oncol 1999;10(suppl 2):S31-S38.

60 Eriksson B, Renstrup J, Imam H, Oberg K: High-dose treatment with lanreotide of patients with advanced neuroendocrine gastrointestinal tumors: clinical and biological effects. Ann Oncol 1997;8:1041-1044.

61 Wymenga AN, Eriksson B, Salmela PI, et al: Efficacy and safety of prolonged-release lanreotide in patients with gastrointestinal neuroendocrine tumors and hormone-related symptoms. J Clin Oncol 1999;17:1111.

62 Ducreux M, Ruszniewski P, Chayvialle JA, et al: The antitumoral effect of the long-acting somatostatin analog lanreotide in neuroendocrine tumors. Am J Gastroenterol 2000; 95:3276-3281.

63 Di Bartolomeo M, Bajetta E, Buzzoni R, et al: Clinical efficacy of octreotide in the treatment of metastatic neuroendocrine tumors. A study by the Italian Trials in Medical Oncology Group. Cancer 1996;77:402408.

64 Arnold R, Trautmann ME, Creutzfeldt W, et al: Somatostatin analogue octreotide and inhibition of tumour growth in metastatic endocrine gastroenteropancreatic tumours. Gut 1996;38:430-438.

65 Aparicio T, Ducreux M, Baudin E, et al: Antitumour activity of somatostatin analogues in progressive metastatic neuroendocrine tumours. Eur J Cancer 2001;37:10141019.
66 O’Toole D, Ducreux M, Bommelaer G, et al Treatment of carcinoid syndrome: a prospective crossover evaluation of lanreotide versus octreotide in terms of efficacy, patient acceptability, and tolerance. Cancer 2000; 88:770-776.

67 Ruszniewski P, Ducreux M, Chayvialle JA, et al: Treatment of the carcinoid syndrome with the longacting somatostatin analogue lanreotide: a prospective study in 39 patients. Gut 1996;39:279-283.

68 Tomassetti P, Migliori M, Gullo L: Slow-release lanreotide treatment in endocrine gastrointestinal tumors. Am J Gastroentero 1998;93:1468-1471.

69 Tomassetti P, Migliori M, Corinaldesi R, Gullo L: Treatment of gastroenteropancreatic neuroendocrine tumours with octreotide LAR. Aliment Pharmacol Ther 2000;14: 557-560.

70 Faiss S, Pape UF, Bohmig M, et al: Prospective, randomized, multicenter trial on the antiproliferative effect of lanreotide, interferon alfa, and their combination for therapy of metastatic neuroendocrine gastroenteropancreatic tumors - The International Lanreotide and Interferon Alfa Study Group. J Clin Oncol 2003;21:2689-2696.

71 Rubin J, Ajani J, Schirmer W, et al: Octreotide acetate long-acting formulation versus open-label subcutaneous octreotide acetate in malignant carcinoid syndrome. J Clin Oncol 1999;17:600-606.

72 Ricci S, Antonuzzo A, Galli L, et al: Octreotide acetate long-acting release in patients with metastatic neuroendocrine tumors pretreated with lanreotide. Ann Oncol 2000;11: 1127-1130.

73 Leong WL, Pasieka JL: Regression of metastatic carcinoid tumors with octreotide therapy: two case reports and a review of the literature. J Surg Oncol 2002;79:180187.

74 Saltz L, Trochanowski B, Buckley M, et al: Octreotide as an antineoplastic agent in the treatment of functional and nonfunctional neuroendocrine tumors. Cancer 1993;72: 244-248.

75 Plockinger U, Dienemann D, Quabbe HJ: Gastrointestinal side-effects of octreotide during long-term treatment of acromegaly. J Clin Endocrinol Metab 1990;71:16581662 .

76 Oberg K, Norheim I, Lind E, et al: Treatment of malignant carcinoid tumors with human leukocyte interferon: long-term results. Cancer Treat Rep 1986;70:1297-1304.

77 Valimaki M, Jarvinen H, Salmela P, Sane T, Sjoblom SM, Pelkonen R: Is the treatment of metastatic carcinoid tumor with interferon not as successful as suggested? Cancer 1991; 67:547-549.

78 Doberauer C, Mengelkoch B, Kloke O, Wandl U, Niederle N: Treatment of metastatic carcinoid tumors and the carcinoid syndrome with recombinant interferon- $\alpha$. Acta Oncol 1991;30:603-605.
79 Creutzfeldt W, Bartsch HH, Jacubaschke U, Stockmann F: Treatment of gastrointestinal endocrine tumours with interferon- $\alpha$ and octreotide. Acta Oncol 1991;30:529-535.

80 Moertel CG, Rubin J, Kvols LK: Therapy of metastatic carcinoid tumor and the malignant carcinoid syndrome with recombinant leukocyte A interferon. J Clin Oncol 1989;7: 865-868.

81 Janson ET, Ronnblom L, Ahlstrom H, et al: Treatment with $\alpha$-interferon versus $\alpha$-interferon in combination with streptozocin and doxorubicin in patients with malignant carcinoid tumors: a randomized trial. Ann Oncol 1992;3:635-638.

82 Oberg K, Alm G, Magnusson A, et al: Treatment of malignant carcinoid tumors with recombinant interferon alfa-2b: development of neutralizing interferon antibodies and possible loss of antitumor activity. J Natl Cancer Inst 1989;81:531-535.

83 Smith DB, Scarffe JH, Wagstaff J, Johnston RJ: Phase II trial of rDNA alfa-2b interferon in patients with malignant carcinoid tumor. Cancer Treat Rep 1987;71:1265-1266.

84 Tiensuu Janson EM, Ahlstrom H, Andersson T, Oberg KE: Octreotide and interferon alfa: a new combination for the treatment of malignant carcinoid tumours. Eur J Cancer 1992;28A:1647-1650.

85 Jacobsen MB, Hanssen LE, Kolmannskog F, Schrumpf E, Vatn MH, Bergan A: Interferon- $\alpha_{2 b}$, with or without prior hepatic artery embolization: clinical response and survival in mid-gut carcinoid patients. The Norwegian Carcinoid Study. Scand J Gastroenterol 1995;30:789-796

86 Dirix LY, Vermeulen PB, Fierens H, De Schepper B, Corthouts B, Van Oosterom AT: Long-term results of continuous treatment with recombinant interferon- $\alpha$ in patients with metastatic carcinoid tumors - an antiangiogenic effect? Anticancer Drugs 1996;7: 175-181.

87 Oberg K, Eriksson B: The role of interferons in the management of carcinoid tumors. Acta Oncol 1991;30:519-522.

88 Nold R, Frank M, Kajdan U, Trost U, Klose KJ, Arnold R: Combined treatment of metastatic endocrine tumors of the gastrointestinal tract with octreotide and interferon- $\alpha$ (in German). Z Gastroenterol 1994;32:193197.

89 Kolby L, Persson G, Franzen S, Ahren B: Randomized clinical trial of the effect of interferon- $\alpha$ on survival in patients with disseminated midgut carcinoid tumours. Br J Surg 2003;90:687-693

90 Frank M, Klose KJ, Wied M, Ishaque N, Schade-Brittinger C, Arnold R: Combination therapy with octreotide and $\alpha$-interferon: effect on tumor growth in metastatic endocrine gastroenteropancreatic tumors. Am J Gastroenterol 1999;94:1381-1387.

91 Oberg K: Chemotherapy and biotherapy in the treatment of neuroendocrine tumours. Ann Oncol 2001;12(suppl 2):S111-S114. 
92 Rougier P, Mitry E: Chemotherapy in the treatment of neuroendocrine malignant tumors. Digestion 2000;62(suppl 1):73-78.

93 Pelley RJ, Bukowski RM: Recent advances in systemic therapy for gastrointestinal neuroendocrine tumors. Curr Opin Oncol 1999;11:32-37.

94 De Jong M, Breeman WA, Bernard HF, et al: Therapy of neuroendocrine tumors with radiolabeled somatostatin analogues. Q J Nucl Med 1999;43:356-366.

95 De Jong M, Breeman WA, Bakker WH, et al: Comparison of ${ }^{111}$ In-labeled somatostatin analogues for tumor scintigraphy and radionuclide therapy. Cancer Res 1998;58: 437-441.

96 Stolz B, Smith-Jones P, Albert R, et al: Somatostatin analogues for somatostatin-receptor-mediated radiotherapy of cancer. Digestion 1996;57(suppl 1):17-21.

97 Valkema R, De Jong M, Bakker WH, et al: Phase I study of peptide receptor radionuclide therapy with [In-DTPA]octreotide: the Rotterdam experience. Semin Nucl Med 2002;32:110-122.

98 Waldherr C, Pless M, Maecke HR, Haldemann A, Mueller-Brand J: The clinical value of ${ }^{90} \mathrm{Y}$-DOTA]-D-Phe ${ }^{1}$-Tyr ${ }^{3}$-octreotide $\left({ }^{90} \mathrm{Y}\right.$-DOTATOC) in the treatment of neuroendocrine tumours: a clinical phase II study. Ann Oncol 2001;12:941-945.

99 Waldherr C, Pless M, Maecke HR, et al: Tumor response and clinical benefit in neuroendocrine tumors after $7.4 \mathrm{GBq}^{90} \mathrm{Y}$-DOTATOC. J Nucl Med 2002;43:610-616.

100 Waldherr C, Schumacher T, Maecke HR, et al: Does tumor response depend on the number of treatment sessions at constant injected dose using ${ }^{90} \mathrm{Y}$-DOTATOC in neuroendocrine tumors? Eur J Nucl Med 2002;29(suppl):S100.
101 Virgolini I, Britton K, Buscombe J, Moncayo R, Paganelli G, Riva P: In- and YDOTA-lanreotide: results and implications of the MAURITIUS trial. Semin Nucl Med 2002;32:148-155.

102 Virgolini I, Traub T, Novotny C, et al: Experience with indium-111 and yttrium-90labeled somatostatin analogs. Curr Pharm Des 2002;8:1781-1807.

103 De Jong M, Breeman WA, Bernard BF, et al: Tumor response after $\left[{ }^{90} \mathrm{Y}^{-D O T A}{ }^{0}, \mathrm{Tyr}^{3}\right]$ octreotide radionuclide therapy in a transplantable rat tumor model is dependent on tumor size. J Nucl Med 2001;42:18411846.

104 Chinol M, Bodei L, Cremonesi M, Paganelli G: Receptor-mediated radiotherapy with Y-DOTA-D-Phe-Tyr-octreotide: the experience of the European Institute of Oncology Group. Semin Nucl Med 2002;32. 141-147.

105 Paganelli G, Zoboli S, Cremonesi M, et al: Receptor-mediated radiotherapy with ${ }^{90} \mathrm{Y}$ DOTA-D-Phe ${ }^{1}$ Tyr $^{3}$-octreotide. Eur J Nucl Med 2001;28:426-434

106 Valkema R, Kvols L, Jamar F, et al: Phase study of therapy with ${ }^{90} \mathrm{Y}-\mathrm{SMT} 487$ (OctreoTher) in patients with somatostatin receptor-positive tumors. J Nucl Med 2002; 5(suppl):33P.

107 Bodei L, Cremonesi M, Zoboli S, et al: Receptor-mediated radionuclide therapy with ${ }^{90}$ Y-DOTATOC in association with amino acid infusion: a phase I study. Eur J Nucl Med Mol Imaging 2003;30:207-216.

108 Rolleman EJ, Valkema R, de Jong M, Kooij PP, Krenning EP: Safe and effective inhibition of renal uptake of radiolabelled octreotide by a combination of lysine and arginine. Eur J Nucl Med Mol Imaging 2003;30: 9-15.

109 Bushnell D, O’Dorisio T, Menda Y, et al: Evaluating the clinical effectiveness of ${ }^{90} \mathrm{Y}$ SMT487 in patients with neuroendocrine tumors. J Nucl Med 2003;44:1556-1560.
110 De Jong M, Breeman WA, Bernard BF, et al: $\left.{ }^{[177} \mathrm{Lu}-\mathrm{DOTA}{ }^{0}, \mathrm{Tyr}^{3}\right]$ octreotate for somatostatin receptor-targeted radionuclide therapy. Int J Cancer 2001;92:628-633.

111 Kwekkeboom DJ, Bakker WH, Kooij PP, et al: $\left.\quad{ }^{177} \mathrm{Lu}-\mathrm{DOTA}^{0}, \mathrm{Tyr}^{3}\right]$ octreotate: comparison with $\left[{ }^{111}\right.$ In-DTPA $\left.{ }^{0}\right]$ octreotide in patients. Eur J Nucl Med 2001;28:13191325.

112 De Jong M, Bernhard HF, Breeman WAP, et al: Combination of ${ }^{90} \mathrm{Y}$ - and ${ }^{177} \mathrm{Lu}$-labeled somatostatin analogs in superior for radionuclide therapy compared to ${ }^{90} \mathrm{Y}$ - or ${ }^{177} \mathrm{Lu}$ labeled analogs only. J Nucl Med 2002:123124.

113 Kwekkeboom DJ, Bakker WH, Kam BL, et al: Treatment of patients with gastroenteropancreatic tumours with the novel radiolabelled somatostatin analogue $\left[{ }^{177} \mathrm{Lu}-\right.$ DOTA $^{0}$, Tyr $^{3}$ octreotate. Eur J Nucl Med Mol Imaging 2003;30:417-422.

114 Kwekkeboom DJ, Teunissen JJ, Bakker $\mathrm{WH}$, et al: Radiolabeled somatostatin ana$\log \left[{ }^{177} \mathrm{Lu}\right.$-DOTA $\left.{ }^{0}, \mathrm{Tyr}^{3}\right]$ octreotate in patients with endocrine gastroenteropancreatic tumors. J Clin Oncol 2005;23: 2754-2762.

115 Jacobsen MB, Nitter-Hauge S, Bryde PE, Hanssen LE: Cardiac manifestations in mid-gut carcinoid disease. Eur Heart J 1995; 16:263-268.

116 Gustafsson BI, Tommeras K, Nordrum I, et al: Long-term serotonin administration induces heart valve disease in rats. Circulation 2005;111:1517-1522.

117 Zuetenhorst JM, Korse CM, Bonfrer JM, Bakker RH, Taal BG: Role of natriuretic peptides in the diagnosis and treatment of patients with carcinoid heart disease. Br J Cancer 2004;90:2073-2079. 